\title{
Packing for the germy: the role of histone H4 Ser1 phosphorylation in chromatin compaction and germ cell development
}

\author{
Kristy D. Wendt ${ }^{1}$ and Ali Shilatifard ${ }^{1,2,3}$ \\ ${ }^{1}$ Department of Biochemistry, Saint Louis University School of Medicine, St. Louis, Missouri 63104, USA; ${ }^{2}$ Saint Louis \\ University Cancer Center, Saint Louis University School of Medicine, St. Louis, Missouri 63104, USA
}

Genetic and epigenetic information is passed to the next generation through germ cells. In this issue of Genes and Development, Krishnamoorthy et al. (2006) have elegantly demonstrated that a conserved histone modification, phosphorylation of Ser1 on histone 4 (H4 S1ph) is involved in chromatin compaction during sporulation in yeast, and that it is an evolutionarily conserved mark found during Drosophila melanogaster and mouse germ cell development.

Post-translational modifications of core histones (PTMs) and incorporation of histone variants/replacement proteins during gametogenesis provide an exceptional example of the relationship between chromatin structure and function. In these instances, histones fulfill their more traditional role as structural regulators and DNA packaging proteins, while providing modification- or variant-specific regulation. Significant changes in chromatin architecture are mediated in part by evolutionarily conserved modifications of histone within the nucleosomes, including ubiquitination, methylation, acetylation, and phosphorylation (Workman and Kingston 1998; Shilatifard 2006). These histone modifications serve to alter chromatin structure and thus regulate transcription factor accessibility to chromatin and affect transcriptional readout.

During metazoan gametogenesis, cells incorporate somatic and gamete-specific histone variants, as well as generalized histone modifications into their chromatin prior to replacement by DNA packaging proteins (Lewis et al. 2003). Spermatogenesis in Drosophila requires the differentiation of round spermatids into an elongated, needle-like structure, with a 200-fold drop in nuclear volume. This is accomplished in part by modification of histones, as well as a highly orchestrated succession of histone replacements by sperm-specific histone variants, then by basic transition proteins, and finally protamines (Sassone-Corsi 2002; Govin et al. 2004; Kimmins and

${ }^{3}$ Corresponding author.

E-MAIL shilatia@slu.edu; FAX (314) 977-5737.

Article is online at http://www.genesdev.org/cgi/doi/10.1101/gad.1477706.
Sassone-Corsi 2005). The appearance of these new histone subtypes and specific histone modification patterns and their correlation with genome compaction, sperm function, and increased fertility, comprise a germ cellspecific histone modification pattern that alters chromatin structure during meiosis.

Saccharomyces cerevisae must protect its genome from damage while simultaneously replicating, recombining, and properly segregating haploid genomes to individual spores. In response to nutrient deprivation, diploid S. cerevisae cells undergo meiosis to produce four stable, metabolically inert haploid spores. Of $\sim 6000$ protein-encoding genes in yeast, $>1000$ show significant changes in expression during the four stages of sporulation-early, middle, mid-late, and late (Chu et al. 1998). These stages result from a sequential cascade of transcription factors and a specialized mitogen-activated protein (MAP) kinase signaling pathway. During meiosis there is strong correlation between a gene's expression pattern and its involvement in a particular biological process. Early genes are typically involved in pairing of homologous chromosomes and recombination, middle genes are required for nuclear division and spore formation, and mid-late genes include those necessary for the outer layer of the spore wall (Chu et al. 1998).

The metaphase peak of post-translational phosphorylation of S10 of histone H3 from late G2 to telophase is a hallmark of mitosis and meiosis in yeast and metazoan species, and this particular modification correlates with chromatin condensation (Hsu et al. 2000; Nowak and Corces 2004). However, mutations altering S10 of H3 do not alter mitosis, meiosis, or cell growth, suggesting a possible redundancy of its function (Hsu et al. 2000; Barber et al. 2004).

In this issue of Genes and Development, Krishnamoorthy et al. (2006) re-examined the function of the previously described phosphorylation of S1 of histone $\mathrm{H} 4$ in chromatin compaction during sporulation. They found H4Serlph to be required for proper chromatin compaction during sporulation, and also for gametogenesis. It is significant that this histone modification is evolutionarily conserved, from Drosophila to mammals. 
H4 S1 is phosphorylated during sporulation, and unlike H3 S10ph, appears to be a stable modification

Krishnamoorthy et al. (2006) report a strong increase in H4 S1ph abundance 8-12 h into the sporulation program. However, the phosphorylation pattern for $\mathrm{H} 3 \mathrm{~S} 10$ and $\mathrm{H} 4$ S1 appears to be temporally distinct during sporulation: Within 4 h of starvation, H3 S10 phosphorylation peaks during the early stage of sporulation and then declines; H4 S1ph appears nearing the completion of meiosis I and II and persists thereafter. H4 S1ph levels only decline after the spores germinate. Therefore, it appears that a H3 S10 phosphatase is active in early sporulation, and that a H4 S1 phosphatase activity is absent during sporulation and becomes activated only once the spores germinate.

Although H3 S10 is a common mitotic/meiotic histone phosphorylation mark correlated with chromatin condensation, mutations that alter H3 S10 do not effect either mitosis or meiosis (Barber et al. 2004). Therefore, Krishnamoorthy et al. (2006) sought to determine whether an H4 S1A mutation demonstrates defects in sporulation. Diploid cells possessing a substitution of alanine for Serl have defects in sporulation. However, other mutations affecting other residues of $\mathrm{H} 4$ do not affect sporulation, indicating that the defect of the S1A mutant is not due to a nonspecific modification of $\mathrm{H} 4$. In light of these observations, it would be interesting to determine whether the expression of $\mathrm{H} 4$ bearing Asp in place of Ser1 in diploid cells could initiate chromatin compaction associated with sporulation in rich media.

\section{H4 S1 is phosphorylated in an Sps1-dependent manner during sporulation}

Expression of $>150$ genes is induced during middle sporulation (Chu et al. 1998). The products of these genes are involved in the control or mechanics of meiotic division, including chromatin compaction. Genes encoding two Ser/Thr protein kinases, Smk1 and Sps1, are among these middle sporulation genes, and Krishnamoorthy et al. (2006) wondered whether these protein kinases are required for H4 S1 phosphorylation. They elegantly demonstrated SPS1 but not SMK1 is required for phosphorylation of H4 S1during sporulation. Furthermore, it appears that Sps1 levels peak at the middle stage of sporulation (similar to the time of appearance for $\mathrm{H} 4 \mathrm{~S} 1 \mathrm{ph}$ ) and decrease thereafter. Since H4 S1ph levels persist even after the disappearance of Sps1, Krishnamoorthy et al. propose that H4 S1ph may be a stable mark required for proper chromatin compaction throughout sporulation.

Although deletion of SPS1 results in the loss of $\mathrm{H} 4$ S1ph during sporulation, Krishnamoorthy et al. (2006) have been unable to demonstrate phosphorylation of $\mathrm{H} 4$ by Sps1 (expressed in bacterial cells and purified) in vitro. Therefore, Sps1 may not be the protein kinase that directly catalyzes phosphorylation of $\mathrm{H} 4 \mathrm{~S} 1$. On the other hand, it is possible that Sps1 needs to be activated via an unknown post-translational modification(s) or accessory protein during middle sporulation to be able to catalyze phosphorylation of H4 S1. Such regulation is a common phenomenon in chromatin biology. Other chromatinmodifying enzymes such as Rad6/Brel require auxiliary factors (Paf1 complex, Bur1/Bur2, etc.) for their function (Krogan et al. 2003; Wood et al. 2003a,b, 2005; Schneider et al. 2005). Furthermore, it is plausible that other posttranslational modifications of chromatin are a prerequisite for phosphorylation of H4 S1 by Sps1. Such regulatory cross-talk is also well established in the chromatin field (Dover et al. 2002; Sun and Allis 2002; Wood et al. 2003a,b; Shilatifard 2006). Determination of essential factors required for phosphorylation of $\mathrm{H} 4 \mathrm{~S} 1$ in response to sporulation will shed further light on the molecular mechanism of this process and define whether Sps1 can directly phosphorylate H4 S1.

\section{Phosphorylation of $\mathrm{H} 4$ S1 can regulate nuclear volume, chromatin compaction, and accessibility}

Although the Western blotting of bulk histones revealed that H4 S1ph is a stable mark during sporulation, chromatin immunoprecipitation studies demonstrated that H4 S1 abundance on chromosomes peaks within $10 \mathrm{~h}$ and declines by $24 \mathrm{~h}$ of sporulation. Both Sps 1 and H4 S1ph appear to have a broad pattern of localization on chromatin, and are enriched at sporulation-specific genes and at many other genomic loci (Krishnamoorthy et al. 2006). However, their abundance consistently decreased as cells traveled farther into the sporulation program. To elucidate the discrepancy between the Western and chromatin immunoprecipitation (ChIP) data regarding the stability of H4 S1ph, Krishnamoorthy et al. (2006) tested $\mathrm{H} 3$ and $\mathrm{H} 4$ abundance on chromatin in wild-type and sps1-null strains to determine whether loss of $\mathrm{H} 4$ S1ph in ChIP studies may be due to inaccessibility resulting from chromatin compaction. Their study indicated that in Sps1's absence, both H3 and H4 ChIP signals are higher than in the wild type, suggesting that $\mathrm{H} 4$ S1ph loss can reduce chromatin compaction and concomitantly histone accessibility. This observation is further substantiated by the observation of increased nuclear volume in the absence of either Sps1 or H4 S1A substitution (Krishnamoorthy et al. 2006).

\section{H4 S1ph is a conserved histone modification of metazoans}

H4 S1ph is also present during spermatogenesis in Drosophila melanogaster as well as mouse cells (Krishnamoorthy et al. 2006). The differential staining pattern for both H4 S1ph and H3 S10ph in Drosophila germ cells parallels that of yeast cells undergoing sporulation. H4 S1ph stains Drosophila chromosomes robustly from prophase to telophase both in meiosis I and II. Although H3 S10ph staining also appears on these chromosomes, peaking at metaphase, its level is substantially reduced in anaphase and telophase. These observations support a model in which the phosphatase that acts on H3 S10ph is active during early to mid stages of sporulation/sper- 
matogenesis and is inactive during spore/sperm development. In Drosophila, H4 S1ph levels persist in spermatid nuclei and are detected in nuclei undergoing chromatin compaction, but are absent in mature spermatids. The stability of H4 S1ph may indicate that this modification is a prerequisite for the highly orchestrated succession of histone replacements by sperm-specific histone variants, then by basic transition proteins, and protamines. Analysis of the role of several Drosophila homologs of Sps1 and other Ser/Thr kinases required for proper spermatogenesis as well as their mammalian counterparts promises to shed further light on the functional conservation of the role of $\mathrm{H} 4 \mathrm{~S} 1 \mathrm{ph}$ in chromatin compaction and germ cell development.

\section{Possible mechanisms for the role of $\mathrm{H} 4 \mathrm{S1ph}$ in chromatin compaction during sporulation and spermatogenesis}

Phosphorylation of core and sperm-specific histones and transition proteins have long been known to occur during spermatogenesis (Sung and Dixon 1970). However, the biological significance for post-translational modifications remains elusive. Krishnamoorthy et al. (2006) propose that $\mathrm{H} 4 \mathrm{~S} 1 \mathrm{ph}$ may function in chromatin compaction. Recent studies from several laboratories have focused on determining the ability of histone modifications to regulate the binding of nonhistone proteins to chromatin and therefore regulate chromatin structure and compaction. This will additionally regulate transcription by modulating interactions between basal transcription machinery and RNA polymerase II with chromatin. However, it was not clear until now that a single histone modification, in the absence of interacting proteins, could play a direct role in regulating chromatin compaction. In their crystal structural studies, Richmond and colleagues (Luger et al. 1997) proposed several years ago that residues 14-23 of the H4 tail are especially important for the formation of $30-\mathrm{nm}$ chromatin fibers, and within this sequence, Lys16 of $\mathrm{H} 4$ is acetylated (Carmen et al. 2002). Stimulated by this observation, Peterson and colleagues (Shogren-Knaak et al. 2006) tested whether a single histone modification might by itself function in the regulation of higher order chromatin structure and provide a potential mechanism to regulate chromatin folding directly via a histone modification. Using a native chemical ligation strategy, Peterson and colleagues (Shogren-Knaak et al. 2006) generated nucleosomal arrays homogeneously acetylated at H4 K16, and showed that this histone modification inhibits formation of compact $30-\mathrm{nm}$ fibers.

Additional support for a direct role for acetylation of $\mathrm{H} 4 \mathrm{~K} 16$ in chromatin decondensation is the observation that H4 K16Ac is highly acetylated in budding yeast, whose genome exists largely in a decondensed state (Lohr et al. 1977; Smith et al. 2003). Since H4 K16Ac is involved in the regulation of chromatin condensation, could other histone modifications regulate chromatin folding as well? This does not appear to be the case for several well-characterized histone modifications, such as H3 S10ph (Fry et al. 2004), triacetylation of the H3 tail by Gcn5p, and residues 1-13 of histone H4 (ShogrenKnaak et al. 2006). These observations suggest that a simple charge modification on a histone tail is not sufficient to regulate chromatin condensation.

The demonstration that phosphorylation of H4 S1 catalyzed by Sps1 can regulate chromatin compaction during sporulation significantly extends our knowledge of the multifaceted roles of histone modifications. Several mechanisms for $\mathrm{H} 4 \mathrm{~S} 1 \mathrm{ph}$ in chromatin compaction can be proposed. First, similar to H4 K16Ac, H4 S1ph may be directly involved in the regulation of chromatin compaction (Fig. 1A). In fact, crystallographic studies with yeast mononucleosomes reveal interaction between the $\mathrm{H} 4$ tail and DNA of a neighboring particle, suggesting a possible direct role for the $\mathrm{H} 4$ tail in the regulation of chromatin compaction (Suto et al. 2000; Zheng and Hayes 2003). Furthermore, it was also demonstrated that the N-terminal tails of histones could play a direct role in chromatin and folding of chromosomes into higher order structures (Moore and Ausio 1997; Carruthers and Hansen 2000). The development of nucleosomal arrays homogeneously phosphorylated at Serl of $\mathrm{H} 4$ and the study of its impact on the formation of compact $30-\mathrm{nm}$ or higher-order fibers should shed further light on this model.

A second probable mechanism for the role of $\mathrm{H} 4 \mathrm{~S} 1 \mathrm{ph}$ in chromatin compaction could be explained by the abil-

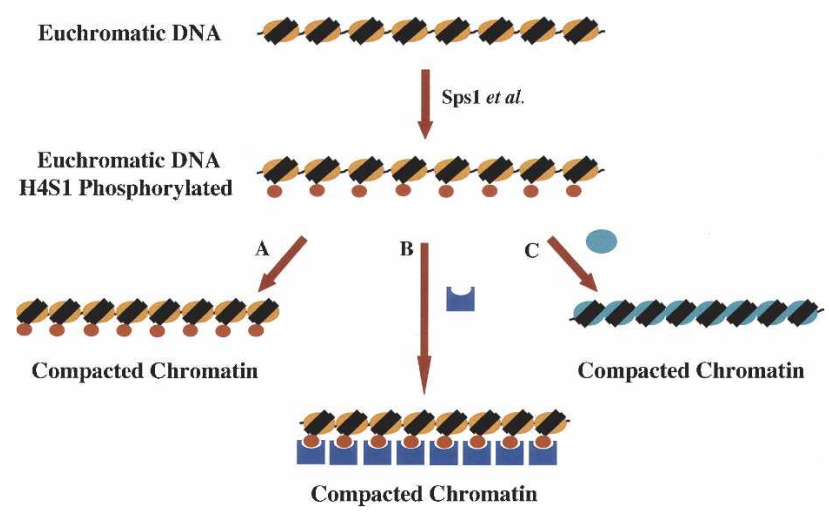

Figure 1. Proposed models for the mechanisms of chromatin compaction during sporulation by phosphorylation of H4 S1. Within 8-12 h into the sporulation program, Serl of histone H4 is phosphorylated in an Sps1-dependent manner. Although Sps1 levels peaks at the middle stage of sporulation similar to the time of appearance for $\mathrm{H} 4 \mathrm{~S} 1 \mathrm{ph}$, the level of Sps1 decreases thereafter. Given this observation, H4 S1ph is considered to be a stable mark throughout sporulation. Several mechanisms for $\mathrm{H} 4 \mathrm{~S} 1 \mathrm{ph}$ in chromatin compaction can be proposed. (A) Similar to $\mathrm{H} 4 \mathrm{~K} 16 \mathrm{Ac}$, H4 S1ph may be directly involved in the regulation of chromatin compaction. (B) Phosphorylated H4 S1 could recruit unknown factor(s) to chromatin that could facilitate chromatin compaction. This is very similar to HP1's role in the formation of heterochromatin via its interaction with methylated H3 K9. (C) Phosphorylated H4 S1 could also play a role as a prerequisite signal for histone replacements by sperm-specific histone variants and/or protamines in metazoans. 
ity of phosphorylated $\mathrm{H} 4 \mathrm{~S} 1$ to specifically recruit factor(s) to chromatin that would further enhance chromatin compaction (Fig. 1B). This would be very similar to the role of HP1 and its interaction with methylated $\mathrm{H} 3$ K9 in the formation of heterochromatin (Nakayama et al. 2001; Elgin and Grewal 2003; Shilatifard 2006). Biochemical and genetic studies defining the existence of possible H4 S1ph-interacting protein(s) should be very informative. Furthermore, since $\mathrm{H} 4 \mathrm{~S} 1 \mathrm{ph}$ seems to be spread throughout the genome and is enriched in nuclei undergoing chromatin compaction and persists in metazoan spermatid nuclei but is lost in mature spermatids, a third possible mechanism for the role of $\mathrm{H} 4 \mathrm{~S} 1 \mathrm{ph}$ in chromatin compaction could be that it plays a role as a signal for the highly orchestrated succession of histone replacements by sperm-specific histone variants and protamines (Fig. 1C).

The study of Krishnamoorthy et al. (2006) is a major breakthrough because it is the first direct correlation between $\mathrm{H} 4 \mathrm{~S} 1 \mathrm{ph}$ and chromatin compaction during spermatogenesis. Future studies defining the molecular role of factor(s) required for specific phosphorylation of this residue of histone $\mathrm{H} 4$ during sporulation and the elucidation of how this modification leads to chromatin compaction will further our understanding of the diverse molecular roles of histones and their modifications in development and differentiation.

\section{Acknowledgments}

We thank Drs. Mark Johnston and Mike Bulger for critical review of this perspective. The work in A.S.'s laboratory is supported by grants from the National Institutes of Health (2R01CA089455 and 1R01GM069905).

\section{References}

Barber, C.M., Turner, F.B., Wang, Y., Hagstrom, K., Taverna, S.D., Mollah, S., Ueberheide, B., Meyer, B.J., Hunt, D.F., Cheung, P., et al. 2004. The enhancement of histone $\mathrm{H} 4$ and $\mathrm{H} 2 \mathrm{~A}$ serine 1 phosphorylation during mitosis and S-phase is evolutionarily conserved. Chromosoma 112: 360-371.

Carmen, A.A., Milne, L., and Grunstein, M. 2002. Acetylation of the yeast histone $\mathrm{H} 4 \mathrm{~N}$ terminus regulates its binding to heterochromatin protein SIR3. J. Biol. Chem. 277: 47784781.

Carruthers, L.M. and Hansen, J.C. 2000. The core histone N termini function independently of linker histones during chromatin condensation. J. Biol. Chem. 275: 37285-37290.

Chu, S., DeRisi, J., Eisen, M., Mulholland, J., Bostein, D., Brown, P.O., and Herskowitz, I. 1998. The transcriptional program of sporulation in budding yeast. Science 282: 699-705.

Dover, J., Schneider, J., Tawiah-Boating, M.A., Wood, A., Dean, K., Johnston, M., and Shilatifard, A. 2002. Methylation of histone $\mathrm{H} 3$ by COMPASS requires ubiquitination of histone H2B by Rad6. J. Biol. Chem. 277: 28368-28371.

Elgin, S.C. and Grewal, S.I. 2003. Heterochromatin: Silence is golden. Curr. Biol. 13: 895-898.

Fry, C.J., Shogren-Knaak, M.A., and Peterson, C.L. 2004. Histone $\mathrm{H} 3$ amino-terminal tail phosphorylation and acetyla- tion: Synergistic or independent transcriptional regulatory marks. Cold Spring Harb. Symp. Quant. Biol. 69: 219-226.

Govin, J., Caron, C., Lestrat, C., Rousseaux, S., and Khochbin, S. 2004. The role of histones in chromatin remodeling during mammalian spermatogenesis. Eur. J. Biochem. 271: 34593469.

Hsu, J.Y., Sun, Z.W., Li, X., Reuben, M., Tatchell, K., Bishop, D.K., Grushcow, J.M., Brame, C.J., Caldwell, J.A., Hunt, D.F., et al. 2000. Mitotic phosphorylation of histone $\mathrm{H} 3$ is governed by Ipl1/aurora kinase and Glc7/PP1 phosphatase in budding yeast and nematodes. Cell 102: 279-291.

Kimmins, S. and Sassone-Corsi, P. 2005. Chromatin remodeling and epigenetic features of germ cells. Nature 434: 583-589.

Krishnamoorthy, T., Chen, X., Govin, J., Cheung, W.L., Dorsey, J., Schindler, K., Winter, E., Allis, C.D., Guacci, V., Khochbin, S., et al. 2006. Phosphorylation of histone H4 Ser1 regulates sporulation in yeast and is conserved in fly and mouse spermatogenesis. Genes \& Dev. (this issue).

Krogan, N.J., Dover, J., Wood, A., Schneider, J., Heidt, J., Boateng, M.A., Dean, K., Ryan, O.W., Golshani, A., Johnston, M., et al. 2003. The Paf1 complex is required for histone H3 methylation by COMPASS and Dotlp: Linking transcriptional elongation to histone methylation. Mol. Cell 11: 721-729.

Lewis, J.D., Abbot, D.W., and Ausió, J. 2003. A haploid affair: Core histone transitions during spermatogenesis. Biochem. Cell Biol. 81: 131-140.

Lohr, D., Kovacic, R.T., and Van Holde, K.E. 1977. Quantitative analysis of the digestion of yeast chromatin by staphylococcal nuclease. Biochemistry 16: 463-471.

Luger, K., Rechsteiner, T.J., Flaus, A.J., Waye, M.M., and Richmond, T.J. 1997. Characterization of nucleosome core particles containing histone proteins made in bacteria. J. Mol. Biol. 272: 301-311.

Moore, S.C. and Ausio, J. 1997. Major role of the histones H3$\mathrm{H} 4$ in the folding of the chromatin fiber. Biochem. Biophys. Res. Commun. 230: 136-139.

Nakayama, J., Rice, J.C., Strahl, B.D., Allis, C.D., and Grewal, S.I. 2001. Role of histone H3 lysine 9 methylation in epigenetic control of heterochromatin assembly. Science 292: $110-113$.

Nowak, S.J. and Corces, V.G. 2004. Phosphorylation of histone H3: A balancing act between chromosome condensation and transcriptional activation. Trends Genet. 20: 214-220.

Sassone-Corsi, P. 2002. Unique chromatin remodeling and transcriptional regulation in spermatogenesis. Science 296: 2176-2178.

Schneider, J., Wood, A., Lee, J.S., Schuster, R., Dueker, J., Maguire, C., Swanson, S.K., Florens, L., Washburn, M.P., and Shilatifard, A. 2005. Molecular regulation of histone H3 trimethylation by COMPASS and the regulation of gene expression. Mol. Cell 19: 849-856.

Shilatifard, A. 2006. Chromatin modifications by methylation and ubiquitination: Implications in the regulation of gene expression. Annu. Rev. Biochem. 75: 243-269.

Shogren-Knaak, M., Ishii, H., Sun, J.M., Pazin, M.J., Davie, J.R., and Peterson, C.L. 2006. Histone H4-K16 acetylation controls chromatin structure and protein interactions. Science 311: 844-847.

Smith, C.M., Gafken, P.R., Zhang, Z., Gottschling, D.E., Smith, J.B., and Smith, D.L. 2003. Mass spectrometric quantification of acetylation at specific lysines within the amino-terminal tail of histone H4. Anal. Biochem. 316: 23-33.

Sun, Z.W. and Allis, C.D. 2002. Ubiquitination of histone H2B regulates $\mathrm{H} 3$ methylation and gene silencing in yeast. $\mathrm{Na}$ ture 418: 104-108. 
Sung, M.T. and Dixon, G.H. 1970. Modification of histones during spermiogenesis in trout: A molecular mechanism for altering histone binding to DNA. Proc. Natl. Acad. Sci. 67: 1616-1623.

Suto, R.K., Clarkson, M.J., Tremethick, D.J., and Luger, K. 2000. Crystal structure of a nucleosome core particle containing the variant histone H2A.Z. Nat. Struct. Biol. 7: 1121-1124.

Wood, A., Schneider, J., Dover, J., Johnston, M., and Shilatifard, A. 2003a. The Pafl complex is essential for histone monoubiquitination by Rad6/Bre1 and histone methylation by COMPASS. J. Biol. Chem. 278: 34739-34742.

Wood, A., Krogan, N.J., Dover, J., Schneider, J., Heidt, J., Boateng, M.A., Dean, K., Golshani, A., Zhang, Y., Greenblatt, J.F., et al. 2003b. Bre1, an E3 ubiquitin ligase required for recruitment and substrate selection of $\operatorname{Rad} 6$ at a promoter. Mol. Cell 11: 267-274.

Wood, A., Schneider, J., Dover, J., Johnston, M., and Shilatifard, A. 2005. The Bur1/Bur2 complex is required for histone H2B monoubiquitination by Rad6/Bre1 and histone methylation by COMPASS. Mol. Cell 20: 589-599.

Workman, J.L. and Kingston, R.E. 1998. Alteration of nucleosome structure as a mechanism of transcriptional regulation. Annu. Rev. Biochem. 67: 545-579.

Zheng, C. and Hayes, J.J. 2003. Intra- and inter-nucleosomal protein-DNA interactions of the core histone tail domains in a model system. J. Biol. Chem. 278: 24217-24224. 


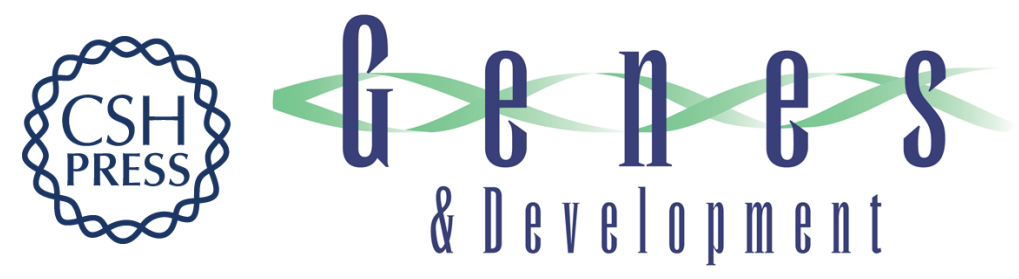

\section{Packing for the germy: the role of histone H4 Ser1 phosphorylation in chromatin compaction and germ cell development}

Kristy D. Wendt and Ali Shilatifard

Genes Dev. 2006, 20:

Access the most recent version at doi:10.1101/gad.1477706 $\begin{array}{cl}\text { Related Content } & \begin{array}{l}\text { Phosphorylation of histone H4 Ser1 regulates sporulation in yeast and is conserved } \\ \text { in fly and mouse spermatogenesis } \\ \text { Thanuja Krishnamoorthy, Xin Chen, Jerome Govin, et al. } \\ \text { Genes Dev. September, 2006 20: 2580-2592 }\end{array} \\ \text { References } & \begin{array}{l}\text { This article cites } 29 \text { articles, } 9 \text { of which can be accessed free at: } \\ \text { http://genesdev.cshlp.org/content/20/18/2487.full.html\#ref-list-1 } \\ \text { Articles cited in: } \\ \text { http://genesdev.cshlp.org/content/20/18/2487.full.html\#related-urls }\end{array} \\ \text { License } & \begin{array}{l}\text { Receive free email alerts when new articles cite this article - sign up in the box at the top } \\ \text { right corner of the article or click here. }\end{array}\end{array}$

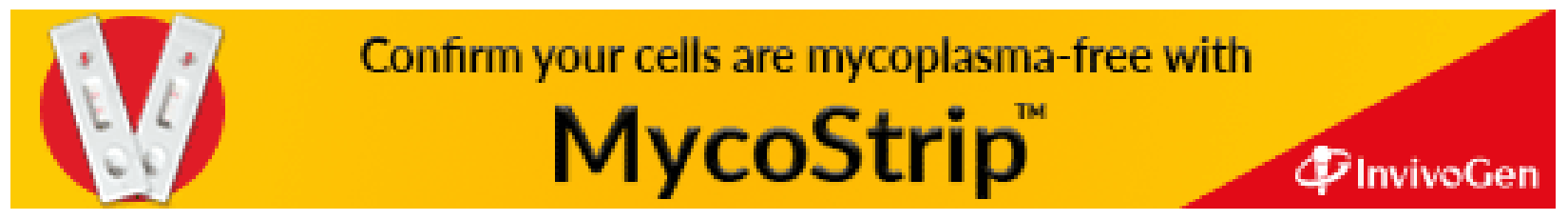

